\title{
O FUNCIONAMENTO DAS UBS - UNIDADES BÁSICAS DE SAÚDE À LUZ CIENTÍFICA DAS FUNÇÕES ADMINISTRATIVAS DE HENRI FAYOL
}

\author{
Mark Wilson da Silva Teixeira ${ }^{1}$ \\ Universidade Estadual de Santa Cruz \\ markwilsonadm@gmail.com \\ https://orcid.org/0000-0002-3584-5017 \\ Prof. Msc. Rozilton Sales Ribeiro ${ }^{2}$ \\ Universidade Estadual de Santa Cruz \\ rosilton@uesc.br \\ ORCID: http://orcid.org/0000-0001-8811-9345 \\ Profa. MSc. Clemilda Gonzaga dos Santos ${ }^{3}$ \\ Universidade Estadual de Santa Cruz \\ clemildags@gmail.com \\ https://orcid.org/0000-0001-7363-3587 \\ Solange Rodrigues dos Santos Corrêa ${ }^{4}$ \\ Universidade Estadual de Santa Cruz \\ srscorrea@uesc.br

Para citar este artículo puede utilizar el siguiente formato:

Mark Wilson da Silva Teixeira, Rozilton Sales Ribeiro, Clemilda Gonzaga dos Santos y Solange Rodrigues dos Santos Corrêa: "O funcionamento das UBS - unidades básicas de saúde à luz científica das funções administrativas de Henri Fayol", Revista Caribeña de Ciencias Sociales (vol 10, № 7 julio-septiembre 2021, pp. 12-24. En línea:

https://www.eumed.net/es/revistas/caribena/julio-septiembre-2021/unidades-basicas-saude

\section{RESUMO}

O presente artigo versou um tema de crucial importância, que é senão, pesquisar as funções administrativas de Henri Fayol (planejar, organizar, coordenar, comandar e controlar) como subsídios e instrumentos de gestão ao gerenciamento das UBS - Unidades Básicas de Saúde. Teve como objetivo geral conhecer o funcionamento operacional destas unidades à luz das funções administrativas de Henri Fayol. E como objetivos específicos, verificar os processos internos básicos no seu funcionamento; discutir as principais causas dos possíveis gargalos no seu fluxo produtivo

\footnotetext{
${ }^{1}$ Pós graduando em Gestão Pública Municipal (Ead)

Curso de Especialização Ead em Gestão Pública Municipal - Pólo de Itabuna 2017. O presente trabalho foi realizado com apoio da Coordenação de Aperfeiçoamento de Pessoal de Nível Superior Brasil (CAPES) - Código de Financiamento 001.

${ }^{2}$ Mestrado em administração pela Universidade Federal da Bahia

${ }^{3}$ Mestrado em administração pela Universidade Federal da Bahia

${ }^{4}$ Mestrado em Desenvolvimento Regional e Meio Ambiente pela Universidade Estadual de Santa Cruz, Doutorado em Desenvolvimento, Agricultura e Sociedade, pela Universidade Federal Rural do Rio de Janeiro
} 
operacional; otimizar o desempenho de suas funções administrativas. Foram constatadas deficiências no processo produtivo principalmente por uma discrepância do sistema ao definir que o profissional de enfermagem exerça funções como assistente à saúde e concomitantemente como administrador mesmo não possuindo conhecimento específico para o gerenciamento. Para tanto, necessário se fez que no referido trabalho se realizasse uma análise do fluxo dos processos internos no atendimento da saúde básica, através da literatura especializada, tornando a pesquisa uma revisão de literatura.

Palavras chave: Atendimento. Eficiência. Funções Administrativas. Gestão em Saúde.

\section{EL FUNCIONAMIENTO DE UBS - UNIDADES BÁSICAS DE SALUD EN LA LUZ CIENTÍFICA DE LAS FUNCIONES ADMINISTRATIVAS DE HENRI FAYOL}

\section{RESUMEN}

El presente artículo abordó un tema de crucial importancia, que es, si no, investigar las funciones administrativas de Henri Fayol (planificar, organizar, coordinar, comandar y controlar) como subvenciones y herramientas de gestión para la gestión de UBS - Unidades Básicas de Salud. Objetivo general conocer el funcionamiento operativo de estas unidades a la luz de las funciones administrativas de Henri Fayol. Y como objetivos específicos, comprobar los procesos internos básicos en su funcionamiento; discutir las principales causas de posibles cuellos de botella en su flujo de producción operativa; optimizar el desempeño de sus funciones administrativas. Las deficiencias en el proceso de producción se encontraron principalmente por una discrepancia en el sistema al definir que el profesional de enfermería desempeña funciones como asistente de salud y concurrentemente como administrador, aunque no tiene conocimientos específicos para la gestión. Para ello, fue necesario que en este trabajo se realizara un análisis del flujo de procesos internos en la atención básica de salud, a través de literatura especializada, haciendo de la investigación una revisión de la literatura.

Palabras clave: Asistencia. Eficiencia. Funciones administrativas. Manejo de la salud.

\section{THE FUNCTIONING OF UBS - BASIC HEALTH UNITS IN THE SCIENTIFIC LIGHT OF HENRI FAYOL'S ADMINISTRATIVE FUNCTIONS}

\section{ABSTRACT}

This article is about a crucial topic, which is to investigate the administrative functions of Henri Fayol (plan, organize, coordinate, command and control) as subsidies and management tools for the management of the Basic Health Units. General objective is to know the operational functioning of these units in the light of the administrative functions of Henri Fayol. And as specific objectives, check the basic internal processes in their operation; discuss the main causes of possible bottlenecks in their operational production flow; optimize the performance of your administrative functions. Deficiencies in the production process were observed, mainly due to a discrepancy in the system, defining that the nursing professional performs functions as a health assistant and concomitantly as 
an administrator, even though he does not have specific management knowledge. To do so, it was necessary that in the mentioned work an analysis of the flow of the internal processes in the basic health care was carried out, through the specialized literature, making the research a review of the literature.

Keywords: Service, Efficiency, Administrative functions, Health Management.

\section{INTRODUÇÃO}

O mundo das organizações sejam elas privadas ou públicas, exige delas alguma competitividade para atender as demandas ocorrentes. Para tal faz-se necessário ações planejadas, bem como uma constante busca pela eficiência interna de suas tarefas. Em seus primórdios, a administração científica preceitua através das funções administrativas de Fayol (1976) esta eficiência, em sua abordagem na teoria clássica da administração. Inicialmente advindas do setor fabril durante a revolução industrial, cada vez mais vem sendo introduzidas no setor público com suas devidas adequações, e no Brasil, através da chamada administração pública gerencial, onde se focam resultados.

Nos primórdios na abordagem clássica da gestão administrativa, Fayol (1976) identifica princípios e funções para as corporações que são difundidas até a nossa atualidade, e dentre estas, as funções administrativas (prever, organizar, comandar, coordenar e controlar) atribuídas ao responsável pelo gerenciamento, que serão o escopo do presente estudo.

A primeira das funções é a de prever, que visa planejar, estabelecer metas e objetivos, diagnosticar e buscar alternativas de ações para resoluções de problemas, no alcance destes objetivos préestabelecidos. Na função organizar dá uma conotação de se constituir os recursos materiais e humanos de forma unívoca na estrutura organizacional. A função comandar significa dirigir as pessoas no sentido dos seus objetivos pertinentes. A função coordenar vislumbra uma união de atos e esforços conjuntos. A função controlar é onde se observa se as regras e os objetivos préestabelecidos estão em conformidade durante sua execução.

A presente investigação pesquisou as funções administrativas de Fayol (1976), enquanto subsídios como instrumentos de gestão ao gerenciamento das UBS - Unidades Básicas de Saúde no SUS Sistema Único de Saúde no Brasil que sugeriu como hipótese estas funções estar ou não sendo observadas adequadamente. E aí surge a problemática: as funções administrativas de Fayol (1976) estarão sendo utilizadas de modo eficiente para agregar valor aos serviços públicos prestados nas UBS - Unidades Básicas de Saúde do SUS - do Sistema Único de Saúde em nossa atualidade?

O objetivo geral nesta investigação foi conhecer o funcionamento operacional das UBS - Unidades Básicas de Saúde à luz das funções administrativas de Fayol (1976). E como objetivos específicos, verificar os processos internos básicos no funcionamento das UBS - Unidades Básicas de Saúde; discutir as principais causas dos possíveis gargalos no fluxo produtivo operacional das UBS; e otimizar o desempenho das funções administrativas nas UBS. 
Justificando a pesquisa, nota-se que a administração científica é à base do que até hoje se formula no gerenciamento de todas e quaisquer corporações, inclusive aos do setor público, neste caso específico, às unidades de saúde. As funções de planejar, organizar, coordenar, comandar e controlar são vivas e presentes, inerentes em todas as organizações públicas, uma vez que a demanda do serviço básico de saúde pública da família é abundante, e aos seus usuários deve-se dar uma resposta sempre positiva na entrega destes serviços. Este estudo se propõe contribuir a um olhar para novos horizontes da administração pública, pois objetiva uma análise conjuntural das funções administrativas de Fayol (1976) através dos processos internos e torná-las visíveis aos gestores mediante pressupostas alternativas de soluções para os problemas das interrupções nos fluxos dos processos que são intrínsecas à eficácia. Assim, é relevante este tomar do conhecimento acerca do assunto para uma melhoria contínua do desempenho nas referidas unidades de saúde.

Este trabalho teve como metodologia aplicada uma revisão de literatura. Para isto, foram utilizadas pesquisas bibliográfica e eletrônica para ora comprovar, ora refutar uma suposta hipótese de que as funções administrativas precedidas por Fayol (1976) podem não estar sendo adequadamente consideradas. Teve uma abordagem bibliográfica-documental-descritiva, e para seu maior enriquecimento considerou como motivação básica a solução de problemas concretos, práticos e operacionais (Zanella, 2009). Então, podemos dizer que é uma pesquisa qualitativa descritiva, preocupada com os processos que permeiam o objeto do presente estudo.

Na secção 1 temos a presente introdução. Na secção 2 está o referencial teórico onde se subdivide em três subitens. No subitem 2.1 estão abordadas as funções administrativas de Henri Fayol no contexto das UBS - Unidades Básicas de Saúde. No subitem 2.2 verifica-se os processos internos no funcionamento das UBS e suas deficiências; e no subitem 2.3 são discutidos alguns gargalos e alternativas para otimizar o desempenho nas UBS. Na secção 3 estão apresentadas as considerações finais.

\section{REFERENCIAL TEÓRICO}

\subsection{AS FUNÇÕES ADMINISTRATIVAS DE HENRI FAYOL NO CONTEXTO DAS UBS}

As funções administrativas foram propostas por Fayol (1976) na abordagem clássica da administração científica para melhor compreensão de uma estrutura organizacional. Advindo do setor privado, com a evolução das teorias administrativas, este conhecimento chega ao setor público, primeiro na Europa e nos Estados Unidos, e depois no Brasil através do modelo da administração pública gerencial uma vez que se fazia necessário uma maior competitividade e eficiência para as organizações públicas (Paludo, 2010).

No Brasil, a saúde pública teve seus primeiros contatos com as funções administrativas de Fayol antes de 1965 no Estado da Guanabara, e, através do decreto-lei ํo 200/67, foram estabelecidas pelo governo brasileiro as bases e as diretrizes de uma reforma administrativa tendo como princípios fundamentais o planejamento, a coordenação, a descentralização (organização), a delegação de competências (comando) e o controle (Campos, 1997). Aqui está a origem da base legal dos 
princípios que norteiam o atual gerenciamento das unidades de saúde no programa da atenção básica.

Para Santos \& Miranda (2007) a atenção básica é a característica da UBS e que as funções de Fayol são esperadas através dos enfermeiros graduados, que devem estar aptos a gerenciar unidades de saúde. Conceituando cada uma das funções administrativas transcritas do livro Administração Industrial e Geral, do próprio Henri Fayol, temos:

Previsão - "a máxima 'governar é prever' dá uma ideia da importância que se atribui a previsão no mundo dos negócios. É verdade que se a previsão não é toda do governo, é dele, pelo menos, uma parte essencial. Prever, aqui, significa ao mesmo tempo calcular o futuro e prepara-lo; é, desde logo, agir" (Fayol, 1976, 58).

Organização - "organizar uma empresa é dotá-la de tudo que é útil a seu funcionamento: matériasprimas, utensílios, capitais e pessoal". Constituir os organismos material e o social de forma uníssona (Fayol, 1976, 72).

Comando - "constituído o corpo social, é preciso fazê-lo funcionar: eis a missão do comando. Essa missão se reparte entre os diversos chefes da empresa, cada um com os encargos e a responsabilidade de sua unidade" (Fayol, 1976, 118).

Coordenação - "é estabelecer a harmonia entre todos os atos de uma empresa, de maneira a facilitar o seu funcionamento e o seu sucesso" (Fayol 1976, 125).

Controle - "consiste em verificar se tudo corre de acordo com o programa adotado, as ordens dadas e os princípios admitidos. Tem por objetivo assinalar as faltas e os erros, a fim de que se possa repará-los e evitar sua repetição. Aplica-se a tudo: às coisas, às pessoas, aos atos" (Fayol 1976, 129). Funções estas que irão transcorrer ao longo dos anos até nossa atualidade se adaptando às diversas corporações públicas em todas as partes do planeta.

Através da Lei 8.080 de 19 de setembro de 1990 foi criado o SUS - Sistema Único de Saúde, regulamentando o artigo 196 da Constituição Federal de 1988. É um sistema descentralizado que, através da municipalização da prestação dos serviços públicos de saúde, fez surgirem a Política Nacional de Atenção Básica e as Unidades Básicas de Saúde - PNAB. E de acordo com esta Política Nacional de Atenção Básica - PNAB (portaria № 2.488 de 21 de outubro de 2011, 4.3.2.1 $\mathrm{VI)}$ uma das atribuições específicas do enfermeiro é "participar do gerenciamento dos insumos necessários para o adequado funcionamento da UBS. Parece oportuno, para o entendimento da prática de enfermagem, a reflexão dessa prática à luz das teorias da administração" (Kurcgant, 1991, 03). A legislação dá um entendimento legal de que o enfermeiro é possuidor da tomada de decisão como gestor das UBS e, portanto, sujeito a estar aliado ao conhecimento científico da administração.

Supondo que um enfermeiro-chefe passasse parte do dia trabalhando no orçamento (planejamento), tivesse uma reunião com funcionários sobre mudanças no sistema de tratamento dado aos pacientes, de cuidados básicos de enfermagem por enfermagem em equipe (organização), alterasse 
a política de pessoal de forma a incluir turnos de 12 horas (pessoal), tivesse uma reunião para resolver um conflito entre enfermeiros e médicos (coordenação) e entregasse uma avaliação de desempenho a um funcionário (controle). Este enfermeiro-chefe não estaria apenas executando todas as fases do processo de administração, mas cada função tem uma fase de planejamento, implementação e controle. Assim como a prática da enfermagem, cada função de administração requer um plano e uma avaliação (Marquis \& Huston, 1999, 32).

E endossando, "como gestor, ou como profissional da UBS, o enfermeiro, em geral, assume a responsabilidade dos materiais permanentes" (Santos \& Miranda, 2007, 237) além da ciência da administração vir evoluindo na gestão da saúde. Ainda assim, faz-se necessário uma melhor verificação dos procedimentos internos de como se executam as funções administrativas neste atendimento básico à saúde.

\subsection{OS PROCESSOS INTERNOS NO FUNCIONAMENTO DAS UBS E SUAS DEFICIÊNCIAS}

Ao imaginarmos a situação hipotética quando um paciente vai a Unidade Básica de Saúde UBS necessitando de atendimento por um médico especialista, e caso não consiga o desejado acolhimento, este não estará presente nas estatísticas da demanda reprimida, uma vez que existem cotas para a demanda espontânea com quantidades limitadas para tais atendimentos. Apesar do acesso universal ao acolhimento, o sistema ainda não contempla o atendimento da demanda espontânea em sua totalidade à população, criando aí uma demanda reprimida.

Contradizendo a função controle, esta informação não estará disponível oficialmente para uma eficiente previsão da demanda e consequentemente para um eficaz planejamento das unidades de saúde se contrapondo com o direito do cidadão ao acesso universal, igualitário e ordenado, como preceitua a legislação através do decreto 7.508 de 28 de julho de 2011, que regulamenta a Lei 8.080/90, a Constituição Federal de 1988 (no direito à saúde em seu artigo 196) e a PNAB - Política Nacional de Assistência Básica.

Há um acolhimento inicial, identificação da população, humanização do atendimento, através de uma triagem como porta de entrada do sistema (Castro \& Shimazaki, 2006). O que diverge da busca pelo objetivo final que é o atendimento à população de modo geral como sendo direito de todos e um dever do Estado este acesso universal, definidos em nossa Carta Magna.

Neste modelo em que o atendimento na UBS - Unidade Básica de Saúde seja a porta de entrada para o SUS, o ingresso na procura regular é feito de forma inicial por uma triagem executada pelo generalista, que busca a melhor alternativa da assistência disponível para cada caso de enfermidade específica, e assim prescrever um acompanhamento compatível para a epidemiologia (frequência, distribuição e determinantes das causas das doenças) da população, bem como o gerenciamento de toda uma gama de insumos e serviços operacionais que dão sustentáculos a esta assistência (Starfield, 2002). 
Como resultado ocorre, porém, quase sempre um gargalo no atendimento à população quando esta procura as unidades de saúde. Nota-se aqui uma deficiência na função de coordenar, pois somente "após imensas filas, salas e corredores abarrotados, longos períodos de espera, por vezes sem resposta [...] número limitado de consultas médicas, distribuição de senhas por ordem de chegada" (Castro \& Shimazaki, 2006, 11), é que se tem a triagem para o atendimento inicial pelo médico clínico geral, há marcações de consulta aos respectivos médicos especialistas, aos exames pertinentes, nova marcação para o retorno aos especialistas, ao fornecimento dos medicamentos gratuitos do governo federal, e ao acompanhamento para o devido tratamento. Acerca deste ciclo operacional ressaltam que "as atividades realizadas na UBS englobam: pré-recepção, recepção, consulta, programas, procedimentos, pós-consulta, visitas domiciliares, atuação nos conselhos locais [...]" (Castro \& Shimazaki, 2006, 22).

Nesta operacionalidade do sistema, as provisões de medicamentos e insumos básicos também são essenciais para o bom funcionamento das unidades de saúde, e são coordenadas de acordo às necessidades das demandas de cada UBS. Porém, "um dos problemas frequentes nas unidades de atenção primária é a falta de materiais que afeta a atenção à saúde do usuário. Portanto, é importante determinar a causa da falta de materiais" (Santos \& Miranda, 2007, 226). Então, a previsão da demanda destes insumos e medicamentos se constitui como um dos fatores preponderantes componente para o sucesso do programa. Faz parte do conjunto dos processos internos e merece atenção redobrada para evitar possíveis gargalos no fluxo do atendimento da saúde básica, uma vez que poderá ser crucial definir vida ou morte dos usuários do sistema.

$\mathrm{Na}$ busca por esta eficiência, técnicas e ferramentas gerenciais são hoje altamente necessárias no apoio para o bom exercício das funções administrativas precedidas por Fayol (1976), que se desmembram em diversas atividades operacionalizadas pelo corpo funcional e que as informações daí advindas são compartilhadas e ajudam na tomada de decisões para uma maior eficácia dos trabalhos pertinentes ao sistema. As atividades executadas, se bem planejadas, organizadas, coordenadas, comandadas e controladas em sinergia, certamente darão respostas positivas para os objetivos a que se propõe a estrutura organizacional como um todo.

$\mathrm{Na}$ visão sistêmica, contingencial (evoluída da teoria da administração científica de Fayol, entre outros) as atuais ações são adaptadas para uma perspectiva contemporânea e devem ser estendidas de forma integradas do nível estratégico, para o nível tático chegando ao nível operacional até às suas unidades produtivas operacionais, como no caso das Unidades Básicas de Saúde em questão, que têm como produto, o serviço especifico de saúde para cada paciente.

O profissional enfermeiro, no que se refere às suas funções gerenciais, pode estar presente nos três níveis organizacionais, ou seja, no nível estratégico, no nível intermediário e no nível operacional (Santana \& Tahara, 2008). Mas a função de prever, o planejamento em si, está presente nas diversas atividades do enfermeiro. Atividades estas, administrativas ou assistenciais, onde não existem limites nítidos entre o gerenciamento administrativo e o gerenciamento clínico, e que são 
tênues, e o enfermeiro ao fazer as duas atividades nas UBS trazem deficiências em ambos os processos.

"Nas instituições de saúde, a estruturação rigidamente hierarquizada estabelece a subordinação integral de um indivíduo a outro, e de um serviço a outro. A enfermagem como um destes serviços, reproduz na sua estruturação o modelo maior. [...] As pessoas e as relações interpessoais não são devidamente consideradas, e as propostas de trabalho resultam em atividades rotineiras [...] Os desenvolvimentos do pessoal de enfermagem e o do próprio serviço ficam, assim, comprometidos" (Kurcgant, 1991, 07).

Vemos, portanto, que a função comando parece destoar, se sobrepõe, ou estar em uma segunda instância, pois na prática da enfermagem não há uma separação nítida entre as funções assistenciais e as administrativas, elas até se complementam, e essas outras atividades ou tarefas são cotidianas para o profissional de enfermagem, pois este profissional no seu dia-a-dia realiza junto com a sua equipe, funções de Planejamento, Organização, Direção e Controle. A aplicação de cada tarefa está de alguma forma em consonância com o trato administrativo em maior ou menor frequência (Melo, 1996). Então esse é outro ponto a ser considerado, a profissão de o enfermeiro estar em desvio de suas funções originais, direcionado às atividades puramente administrativas, mesmo estando em conjunto com as suas prerrogativas de ações assistenciais.

Contudo, ainda assim não se consegue assegurar o acesso com eficácia e atender às necessidades dos cidadãos (Castro \& Shimazaki, 2006), pois existem conflitos na definição de uma autoridade com poderes de decisão nas UBS, uma vez que a enfermagem que ocupa tais funções se prevalecem da CIPESC - Classificação Internacional das Práticas de Enfermagem em Saúde Coletiva, onde o profissional de enfermagem mesmo não tendo a formação acadêmica científica para a gestão da coisa pública, tem autonomia operacional e na tomada de decisão (Setenta, 2005).

E o que sucede ainda hoje é que os profissionais de saúde, enfermeiros ou médicos, os gerenciadores das tomadas de decisões nas unidades de saúde, tiveram seus estudos direcionados especificamente para a acuidade da saúde, e que pouco ou nada tiveram em seu currículo escolar o conhecimento científico teórico das ciências administrativas para lidar diretamente com a eficácia administrativa, mas que assim o fazem, de forma remediada no dia a dia pelo contato direto com as demais atividades dentro das unidades de saúde.

Além disto (das limitações do conhecimento das ciências administrativas pelo corpo gerencial); a falta de médicos especialistas; cotas limitadas para o atendimento; e a falta de medicamentos e de insumos são alguns dos gargalos frequentes (causados por equívocos na função organizar) que fazem a assistência básica de saúde não atingir seus objetivos, vez que a percepção da população afirma não satisfazer a demanda da clientela abrangente em sua plenitude (Castro \& Shimazaki, 2006).

Vale salientar que "em alguns serviços públicos, a falta de profissionais específicos para esta função faz com que o trabalho seja realizado de forma improvisada. Em alguns casos, é exercido por 
funcionários desviados de sua função. [...] Isso pode levar a inúmeros transtornos, prejudiciais ao abastecimento das unidades de saúde e por consequência ao atendimento à população" (Santos \& Miranda, 2007, 232). Acrescentam ainda que "frequentemente, procedimentos ditos 'da porta de entrada' ficam grandes períodos sem serem realizados, por falta de material, uso inadequado, ou ainda, por falta de conserto (aparelhos de P.A., pinças, gazes e outros). Isso deve ser evitado, pois há risco de que a UBS deixe de cumprir seu papel por gestão inadequada de recursos" (Santos \& Miranda, 2007, 233). E expõem ainda que entre as questões mais preocupantes em relação à falta dos materiais e insumos estão "os medicamentos, os materiais chamados de enfermagem (gazes, pinças, esparadrapos, algodão, soluções e outros), além de material de odontologia" (Santos \& Miranda, 2007, 233).

As constatações destas deficiências aqui observadas podem servir como feedback para proposituras de como sanar ou mitigar as imperfeições do sistema, e assim melhorar seu desempenho.

\subsection{OTIMIZAÇÃO DO DESEMPENHO NAS UBS}

A sociedade cidadã busca uma saúde pública eficiente que deva oferecer um atendimento universal e ilimitado em sua totalidade, mas nas UBS se verificam falhas a serem reparadas para consolidar a eficácia do sistema. O modelo sistêmico permite as organizações reverem sua programação e corrigir possíveis desvios.

Nesta visão como um todo, a estrutura organizacional das UBS se consolida através das entradas (atendimento aos indivíduos), dos processos (atividades programadas), das saídas (resultado do serviço aos pacientes) e do feedback ou retroalimentação (o nível de satisfação da população) como subsídio para melhoria contínua neste ciclo operacional (Santana \& Tahara, 2008). E em nossa atualidade contemporânea, contingencial, do conhecimento, muitas são as tecnologias disponíveis às organizações, sejam públicas ou privadas, que aliadas a técnicas e ferramentas administrativas existentes podem oferecer competitividade na busca por resultados, dizimando estes erros porventura encontrados no sistema.

Há que se ter uma nova mentalidade de gestão dentro da administração pública gerencial, e a reengenharia de Willian Edwards Deming (1900-1993) tornou-se paradigma nesta evolução. Reengenharia é conceituada como "repensar de forma fundamental e reprojetar radicalmente os processos [...] para conseguir melhorias drásticas a serem medidas por índices críticos e contemporâneos de desempenho tais como custos, qualidade, atendimento e velocidade" (Cruz apud Hammer, 1997, 29).

Notadamente, a população espera obter um atendimento integral no que se refere ao acesso à saúde, onde se pretende ter um acolhimento com qualidade satisfatória para a acuidade e tratamento de suas enfermidades. Para tal, existe toda uma cadeia produtiva e uma estrutura mínima para este atendimento de forma diagnosticada, planejada e ordenada para que se concretize dando uma resposta positiva, firmando assim, um bom posicionamento à clientela atendida. 
Corporações que possuam um sistema de atendimento e que pretendam ser eficazes, como no caso das Unidades Básicas de Saúde - UBS, pressupõe-se terem uma eficiente previsão da demanda com o objetivo de subsidiar informações para o planejamento e assim minorar deficiências ou suprir porventura a falta de medicamentos, de insumos e das especialidades médicas à totalidade da população que busca estes serviços.

Não deveriam impor cotas. O atendimento deveria ser fornecido indiscriminadamente, a todos que procurassem os serviços nestas unidades. Uma previsão da demanda através das séries históricas sazonais permitiria um rearranjo na quantidade de especialistas médicos a serem disponibilizados com a filosofia just-in-time que foca nos processos, na produção em tempo de atender necessidades do serviço, eliminando desperdícios (Dias, 1995). O médico especialista adequado no momento certo, na quantidade exata para atender aos pacientes. Os mesmos procedimentos deveriam ser para o fornecimento de medicamentos, limitando-se apenas ao que já foi estipulado por lei, o tipo de medicamentos e a quantidade dentro das limitações orçamentárias.

Em relação aos materiais e insumos "o objetivo básico da administração de materiais consiste em disponibilizar os recursos necessários para a realização do processo produtivo com qualidade, em quantidades adequadas, no tempo correto e com menor custo" (Santos \& Miranda, 2007, 226). E dentre os métodos para renovação de estoques mais usuais estão os modelos de estoque mínimo e de estoque máximo ou renovação periódica. Ou seja, os recursos materiais devem estar sempre à disposição, de forma correta e com menor custo, sendo consideradas técnicas de controles de estoques mínimos e máximos (Santos \& Miranda, 2007, 229).

As séries históricas estatísticas são a ferramenta mais adequada e mais usual para se fazer uma análise do comportamento desta demanda sazonal, e assim ajudar numa reorientação programada as ações governamentais tanto para atender a demanda das especialidades médicas quanto para 0 suprimento de medicamentos gratuitos para a população.

Vale salientar que séries históricas estatísticas onde estas diagnosticam uma demanda reprimida, poderão contribuir no planejamento das organizações em geral bem como se estender como subsídios à gestão no setor público. Elas poderão compor informações das especialidades médicas mais ausentes nos últimos cinco anos e que serão o feedback para um rearranjo organizacional do acesso universal à saúde básica, através de uma vigilância epidemiológica mais eficiente e eficaz.

Outra técnica é a curva $A B C$ de estoque onde se analisam itens com as frequências de seus respectivos eventos e classificam aqueles que mais se aproximam aos objetivos das organizações de acordo com as prioridades em suas peculiaridades (Dias, 1995). Esta é outra técnica que poderá ser adaptada para o estudo da demanda reprimida no atendimento das especialidades médicas procuradas na área de abrangência da UBS, bem como no suprimento dos insumos e medicamentos a serem disponibilizados à procura da população abrangente. 
Quanto aos funcionários gestores, já tanto mencionado aqui, estes deveriam receber uma qualificação em administração uma vez que trabalham diretamente com as funções de planejar, organizar, coordenar, comandar e controlar em seu dia-a-dia nas unidades básicas.

Gestores adequadamente capacitados direcionados para a gestão, previsão da demanda das especialidades médicas e dos materiais e medicamentos através das técnicas de séries históricas estatísticas e da curva $A B C$ de estoque, aliadas a uma eficaz tecnologia de informação - TI são fundamentais para o sucesso da implementação de um planejamento que se predispõe a oferecer um atendimento verdadeiramente integral com alto de grau de satisfação à sua população abrangente da UBS.

Vê-se então que a busca pela melhoria contínua do desempenho no atendimento à saúde básica em suas unidades, processa-se por um moderno modelo de pensar e de agir, através de uma mentalidade proativa e práticas inovadoras visando um diagnóstico da situação adicionada a contribuições como a reestruturação com uma nova ordenação de funções de autoridade e responsabilidade, previsão das demandas das especialidades e insumos, seu suprimento e respectivo controle.

\section{CONSIDERAÇÕES FINAIS}

Neste estudo de pesquisa, através de uma revisão de literatura o que ficou constatado foi que, apesar da legislação dizer que o acesso à saúde seja universal, a análise entre os escrutínios literários de diversos escritores nos afirma que isto é apenas uma falácia, um engodo. O que se presume ser acesso universal nada mais nada menos é do que um acolhimento territorial onde são cadastradas pessoas em determinada área geográfica para um controle da multidão que paga seus impostos e não recebem a contrapartida em forma da saúde universal tão propalada na Constituição Federal e demais leis pertinentes.

Os principais profissionais especialistas responsáveis pela saúde nestas unidades básicas são direcionados quase que exclusivamente à acuidade ao paciente, não tendo uma preparação acadêmica em seu currículo o trato efetivo para com as bases teóricas da administração científica resultando em ineficiência e ineficácia no gerenciamento das unidades de saúde, e consequentemente na qualidade dos serviços de saúde prestados à população.

Com o destrato desta causa pública, faz-se criar, ainda que inconscientemente, através de nossa cultura, uma reserva de mercado para o setor privado. O que denota que os profissionais de saúde, seus colaboradores toda estrutura funcional das UBS, a bem da administração pública deveriam receber uma atenção mais especial por parte dos poderes públicos.

Uma vez que não há médicos suficientes para atender a população em geral, em que não há cotas suficientes para especialidades médicas, em que não há medicamentos e insumos suficientes, apesar da legislação em vigor preceituar este tão propagado direito universal à saúde, sugerimos aos próximos pesquisadores buscarem o aprofundamento deste estudo através de dados mais 
atualizados nos portais da transparência de seus municípios, inclusive com o foco na determinação de que os gestores da saúde pública legitimam uma bilionária reserva de mercado para o setor privado, o que aqui não foi escopo do estudo. Recomendamos também uma análise mais substancial na decomposição dos processos internos para servir como base de um possível manual de operações e atividades detalhadas de formas específicas bem definidas no que diz respeito às tarefas administrativas em cada uma das funções administrativas de Fayol com suas atribuições, limites de autoridade e respectivas responsabilidades. Para que este manual seja transformado em lei.

Respondendo à problemática da pesquisa, constatou-se por diversos autores enunciados, que, como na UBS não existe o cargo específico de gestor, o que causa acúmulo de atribuições para o profissional de enfermagem ao exercer concomitantemente as funções de enfermeiro e de administrador, as funções administrativas preceituadas (Fayol 1976: (planejar, organizar, coordenar, comandar e controlar) não estão sendo utilizadas adequadamente pelos gestores das unidades de saúde, uma vez que não são realizadas sistematicamente de forma integradas.

\section{REFERÊNCIAS}

Brasil (1988). Constituição Federal. Brasília, DF: Câmara dos Deputados.

Campos, J. Q. (1997). Administração de saúde: técnicas de organização. Editora Jotacê.

Castro, A. J. R. \& Shimazaki, M. E. (2006). Protocolos clínicos para unidades básicas de saúde. Escola de Saúde Pública.

Cruz, T. (1997). Manual de organização: reengenharia na prática (2 $2^{\mathrm{a}}$ ed.). Atlas.

Dias, M. A. P. (1995). Administração de materiais: edição compacta (4⿳亠丷a ed.). Atlas.

Fayol, H. (1976).Trad. Irene de Bojano e Mário de Souza. Administração industrial e geral (9a ed.). Atlas.

Hammer, M. \& Champy, J. (1993). Reengineering the corporation. Harper Bussines.

Kurcgant, P. (1991). Administração em enfermagem. EPU.

Marquis, B. L. \& Huston, C. J. (1999). Administração e liderança em enfermagem: teoria e aplicação ( $2^{\mathrm{a}}$ ed.). Artmed.

Melo, M. R. A. C., Fávero, N., Trevizan, M. A. \& Hayashida, M. (1996). Expectativa do administrador hospitalar frente as funções administrativas realizadas pelo enfermeiro. Rev. Latino-Am. Enfermagem, 4(1), 131-44.

Ministério da Saúde (2012). Secretaria de Atenção à Saúde/Departamento de Atenção Básica. Política Nacional de Atenção Básica. Ministério da Saúde.

Ministério da Saúde/Gabinete do Ministro (2011). Portaria nº 2.488/2011 de 21 de outubro. Acessado em agosto de 2018. http://bvsms.saude.gov.br/bvs/saudelegis/gm/2011/prt2488 $21 \quad 10$ 2011.html

Paludo, A. V. (2010). Administração pública: teoria e questões. Elsevier.

Presidência da República (1988). Constituição da República Federativa do Brasil: texto constitucional promulgado em 05 de outubro de 1988, com as alterações adotadas pelas Emendas 
Constitucionais n 1/92 a 56/2007 e pelas Emendas Constitucionais de revisão $n 1$ a 6/94. Senado Federal, Subsecretaria de Edições Técnicas.

Presidência da República, Casa Civil (1990). Lei 8.080/1990 de 19 de setembro. Acessado em agosto de 2018. http://www.planalto.gov.br/ccivil 03/leis/L8080.htm

Santana, R. M. \& Tahara, A. T. S. (2008). Planejamento em enfermagem: aplicação do processo de enfermagem na prática administrativa. Editus.

Santos, A. S. \& Miranda, S. M. R. C. (2007). A enfermagem na gestão em atenção primária à saúde. Manole.

Setenta, E. X. M. \& Mendes, V. L. P. S. (2005). Ações gerenciais da(o)s enfermeira(o)s na reorganização dos serviços de saúde da rede básica: um estudo no município de Itabuna/Ba. Salvador/BA: Universidade Federal da Bahia, Escola de Enfermagem.

Starfield, B. (2002). Atenção primária: equilíbrio entre necessidades de saúde, serviços e tecnologia. UNESCO/MS.

Zanella, L. C. H. (2009). Metodologia de estudo e de pesquisa em administração. Departamento de Ciências da Administração/UFSC; CAPES; UAB. 